\title{
Entwicklung und empirische Validierung eines kontextorientierten Skalenmodells zur Erfassung des Interesses von Schüler*innen am Klimawandel
}

\author{
Bernhard Thieroff' 1 (D) J Jan Christoph Schubert ${ }^{1} \cdot$ Dietmar Gölitz' $^{1}$ \\ Eingegangen: 17. Juli 2020 / Angenommen: 30. Januar 2021 / Online publiziert: 18. Februar 2021 \\ (c) Der/die Autor(en) 2021
}

\section{Zusammenfassung}

Der Klimawandel stellt eine der wesentlichen Herausforderungen des 21. Jahrhunderts dar und gilt durch seine Struktur als hochrelevantes Thema im Geographieunterricht. Gleichzeitig stellt Interesse eine der zentralen themenspezifischen Voraussetzungen für fachliches Lernen dar. Bezogen auf den Klimawandel ist dieses bisher allerdings nur in Ansätzen erforscht. Die vorliegende Studie entwickelte daher auf Basis themenspezifischer sowie geographiedidaktischer Vorüberlegungen einen Fragebogen zur Erfassung kontextbezogenen Schüler*inneninteresses am Klimawandel. Im Zuge einer Faktorenanalyse ergaben sich fünf eindeutige Faktoren mit jeweils angemessenen bis hohen Reliabilitätswerten. Auf Ebene der unterrichtlichen Kontexte zeigten sich die lebensweltlich orientierten und individuell geprägten Kontexte Handlung sowie räumliche Nähe als besonders gewichtige Determinanten des Schüler*inneninteresses. Weiterhin ergab sich der Kontext Messung/Methodik. Mit Blick auf die fachlich-inhaltliche Struktur des Themas konnten die Faktoren globale Ursachen sowie Folgen als interesseleitend identifiziert werden. Es zeigte sich, dass das Interesse am Klimawandel vor allem durch die Lebenswelt sowie den Alltag der Schüler*innen betreffende Kontexte (z. B. den Nah-/Heimatraum) determiniert wird. Die inhaltlichen Facetten des Themas spielen - zumindest soweit diese Kontexte berührt werden - eine nachgeordnete Rolle. Somit liegt ein hochreliables Verfahren zur Erfassung des Schüler*inneninteresses am Klimawandel gegen Ende der Sekundarstufe I vor, das eine Ausdifferenzierung des derzeitigen Forschungsstandes sowie eine interessenbezogene Wirksamkeitsprüfung unterrichtlicher Interventionen ermöglicht.

Schlüsselwörter Klimawandel $\cdot$ Interesse $\cdot$ Kontexte $\cdot$ Lernvoraussetzungen $\cdot$ Skalenentwicklung

Bernhard Thieroff

bernhard.thieroff@fau.de
Lehrstuhl für Didaktik der Geographie, Friedrich-Alexander-Universität Erlangen-Nürnberg, Regensburger Str. 160, 90478 Nürnberg, Deutschland 


\title{
Development and Empirical Validation of a Content-oriented Scale Model to Asess the Students' Interest in Climate Change
}

\begin{abstract}
Climate change is one of the major challenges of the 21 st century and is considered a highly relevant topic in geography education due to its structure. At the same time, interest is one of the central subject-specific preconditions for content learning. In relation to climate change, however, students' interest has not been addressed enough in research so far. The presented study therefore developed a questionnaire for the gathering of context-related students' interest in climate change based on subject-specific and didactic considerations. In the course of a factor analysis, five clear factors emerged, each with appropriate to highly reliable values. At the level of the teaching contexts, the everyday-life oriented and individually shaped contexts Action and Spatial Proximity were found to be particularly important determinants of the students' interest. Furthermore, the context Measurement/Methodology emerged. With regard to the content structure of the topic, the factors Global Causes and Consequences could be identified as factors leading to interest. It could be shown that the interest in climate change is mainly determined by the living environment and the contexts of students' everyday life (e.g. the immediate vicinity/home area). The content-related facets of the topic play a subordinate role - at least as far as the mentioned contexts are concerned. Hence, a highly reliable method for determining students' interest in climate change at the end of secondary school is available, which allows a differentiation of the current state of research and an interest-based assessment of the effectiveness of teaching interventions.
\end{abstract}

Keywords Climate Change $\cdot$ Interest $\cdot$ Contexts $\cdot$ Learning Preconditions $\cdot$ Scale Development

\section{Einleitung}

Der Klimawandel gilt als eine der zentralen Herausforderungen des 21. Jahrhunderts (IPCC 2014; Endlicher und Gerstengarbe 2007). Durch die permanente Interaktion verschiedener Geofaktoren (Borsdorf 2019) handelt es sich dabei um einen komplexen naturwissenschaftlichen Prozess, der auf verschiedenen räumlichen Maßstabsebenen sowie zeitlichen Dimensionen angesiedelt ist. Im schulischen Kontext nimmt das Fach Geographie, das sich als Brückenfach zwischen Natur- und Sozialwissenschaften mit ausgeprägtem systemischen Charakter versteht (DGfG 2017), bei der Behandlung des Themenkreises Klimawandel eine besondere Bedeutung ein.

Lernprozesse werden von verschiedenartigen Bedingungsfaktoren beeinflusst. Im Bereich der individuellen Determinanten spielen neben der Intelligenz als gewichtigstem Prädiktor von Schulleistung auf fach- und themenspezifischer Ebene vor allem das Vorwissen sowie motivationale Faktoren wie Einstellungen oder Interesse eine entscheidende Rolle (Helmke und Schrader 2010). Eine auf Interesse beruhende Lernmotivation gilt dabei als besonders erstrebenswert, da diese „besonders intensive und wirkungsvolle Lernprozesse in Gang setzt“ (Krapp 1992c, S. 41), auch der Zusammenhang zwischen Interesse und Schulleistung ist mit $r=0,30$ durchaus substanziell (Krapp 2010a), andere Autoren nennen sogar $r=0,41$ (Helmke und Schrader 2010; Helmke und Weinert 1997). Der Einfluss von Motivation auf Schulleistung ist ein mittlerer $(d=0,48$; Hattie 2012). Auch wenn Interesse nicht automatisch zu entsprechender Handlung führt, so fördert es im Sinne der Ausbildung einer Scientific Literacy die Bereitschaft, sich anwendungsorientiert mit einer Thematik auseinanderzusetzen (OECD 2006) und ist so ein wesentlicher Bedingungsfaktor klimarelevanter Handlungen (Krapp 1992b; Rheinberg 2010). Obwohl das themenspezifische Interesse am Klimawandel somit eine entscheidende Lernvoraussetzung für einen hochrelevanten Lernbereich darstellt, ist es bisher nur unzulänglich erforscht. Gleichzeitig ist der Klimawandel durch die gesellschaftlichen Entwicklungen der letzten Jahre im Zeichen von Fridays For Future verstärkt in den medialen Fokus sowie an die Lebensrealität der Jugendlichen gerückt. Dies erfordert eine aktuelle und ausdifferenziertere Erforschung des Interesses von Schüler*innen am Themenfeld Klimawandel. Dieser Herausforderung begegnet der vorliegende Beitrag in einem ersten Schritt durch die Vorstellung eines einschlägigen Messinstrumentes.

\section{Theoretische und empirische Grundlegung}

\section{Interesse und Interessensentwicklung}

Im schulischen Anwendungsfeld ist Interesse sowohl als Lernziel (Hemmer et al. 2005) als auch als fachspezifische Lernvoraussetzung und damit verbunden vor allem in Bezug auf Lernmotivation relevant. Krapp (2010a, S. 10) fasst unter Lernmotivation „die Bereitschaft eines Schülers, sich aktiv, dauerhaft und wirkungsvoll mit bestimmten Themengebieten auseinander zu setzen, um neues Wissen zu erwerben bzw. das eigene Fähigkeitsniveau zu verbessern“" und unterscheidet grundsätzlich zwischen sogenannten 
kognitiv-handlungstheoretischen und persönlichkeitstheoretischen Konzepten. Während die auf dem ErwartungsWert-Modell (Rheinberg 2010) fußende kognitiv-handlungstheoretische Konzeption statisch ist und nicht direkt vom Lerngegenstand determiniert wird (Krapp 2010a), haben persönlichkeitstheoretische Konzepte einen deutlich engeren Bezug zu themenspezifischem fachlichen Lernen. Diese betrachten Lernmotivation als an die Persönlichkeit gekoppeltes, dynamisches Konstrukt und fassen Lernen nicht nur als inhaltliche Wissenskonstruktion, sondern integrieren auch die Ausbildung und Veränderung von emotionalen und motivationalen Komponenten (z.B. Einstellungen, Überzeugungen, Werthaltungen; Krapp 2010a), was besonders bei einem persönlichkeits- und handlungsrelevanten Thema wie dem Klimawandel von besonderer Bedeutung ist. Neben der Selbstbestimmungstheorie von Ryan und Deci (2004) hat vor allem die, mit der Selbstbestimmungstheorie verwandte, Person-Gegenstands-Theorie des Interesses (Krapp 1992a, 1992c, 2010a) Bekanntheit erlangt und stellt eine wesentliche Grundlage pädagogischer sowie fachdidaktischer Interessenforschung dar (Krapp 2010a; Hemmer 2010; Häußler et al. 1996; Blankenburg und Scheersoi 2018; Gebhard et al. 2017).

Interesse im Sinne dieser Theorie bezeichnet eine ,bedeutungsmäßig herausgehobene Person-Gegenstands-Relation“ (Krapp 1992a, S. 307), die mit einer ",hohen Bereitschaft zur freiwilligen Beschäftigung“ (Krapp 2010a, S. 15) einhergeht. Der Gegenstand des Interesses ist definitorisch kaum eingegrenzt, es kann sich dabei sowohl um ein konkretes materielles oder immaterielles Objekt, als auch um ein Thema, eine Theorie oder eine Idee handeln, nahezu alles, womit eine kognitive Auseinandersetzung möglich ist. Im schulischen Kontext ist besonders die Unterscheidung zwischen Sach- und Fachinteresse zu erwähnen - wendet eine Person beispielsweise dem Themenkreis Klimawandel besonderes Interesse zu, so liegt hohes Sachinteresse an dieser speziellen Facette des Faches Geographie vor, interessiert sich eine Person allerdings besonders für das (Schul-)Fach Geographie im Allgemeinen (z. B. an der räumlichen Betrachtungsweise von Sachverhalten), so spricht man von hohem Fachinteresse (Krapp 2010a). Definitorisch lässt sich Interesse durch drei Kernmerkmale charakterisieren: „In emotionaler Hinsicht zeichnet sich ein Interesse dadurch aus, dass es an überwiegend positive emotionale Erfahrungen geknüpft ist" (Krapp 2010a, S. 16) - die Beschäftigung mit dem Interessengegenstand wird daher nicht als störend, sondern eher als zufriedenstellend wahrgenommen, die teilweise in einen sogenannten Flow-Zustand (Csikszentmihalyi 1999) übergehen kann. Die wertbezogene Komponente des Interesses betont die subjektive Wichtigkeit des Interessengegenstandes für eine Person. Sich mit dem Interessengegenstand auseinanderzusetzen, um z. B. die eigenen Kompetenzen in einem Bereich zu erweitern, wird demnach als sinnvoll und wichtig empfunden und gilt als Teil der eigenen Identitätsbildung. Die kognitive Komponente des Interesses bezeichnet die Notwendigkeit, ein Mindestmaß an Wissen über den Interessengegenstand zu besitzen, da gänzlich unbekannte Objekte weder als wichtig eingeschätzt werden können, noch kann überhaupt eine (möglicherweise als positiv empfundene) Beschäftigung mit diesen stattfinden (Krapp 2010a).

Blickt man auf die Struktur des Interessenkonstrukts (s. Abb. 1), so kann Interesse als langfristige bzw. dauerhafte Disposition einer Person auftreten (trait). Dieses, einer Person bereits immanente, individuelle Interesse kann im Unterricht durch die Lehrperson aufgegriffen und verstärkt werden und dadurch in ein aktualisiertes (individuelles) Interesse überführt werden (Thomas und Kirby 2020). Wird im Unterricht eine Thematik oder Arbeitsweise behandelt, für die grundsätzlich noch kein positives Interesse existiert, so kann die Lehrkraft durch die Interessantheit der Lernumgebung (bspw. die Materialauswahl oder das Lehrer*innenhandeln) ein vorübergehendes situationales Interesse hervorrufen, das zu erhöhter Lernmotivation (state) führt und in individuelles Interesse übergehen kann (Renninger und Hidi 2019; Krapp 2010a; Vogt 2007; Blankenburg und Scheersoi 2018). Auch im AngebotsNutzungs-Modell (Helmke 2017) wird auf Interesse (bzw. darauf beruhende Lernmotivation) im Sinne der PersonGegenstands-Theorie zum einen als veränderbare, einer Person innewohnende Lernvoraussetzung sowie zum anderen als konstituierendes Merkmal der Lernumgebung aufgefasst, die jeweils die Nutzung des unterrichtlichen Angebots beeinflussen.

Stellt die Person-Gegenstands-Theorie eine Rahmung der Interessenstruktur dar, so lässt sich diese je nach Anwendungsgebiet weiter charakterisieren: Im Bereich der Fachdidaktiken haben beispielsweise Upmeier zu Belzen und Vogt das neben dem Interesse bestehende „Nichtinteresse" weiter in Desinteresse und Abneigung ausdifferenziert, wobei ersteres eher als Gleichgültigkeit und letzteres als Antipathie beschrieben werden kann (Vogt 2007). Im Rahmen der IPN-Interessenstudie wurde das Sachinteresse am Fach Physik noch feiner in die Komponenten Interesse an (1) einem Themengebiet, (2) einem Kontext und (3) ei-

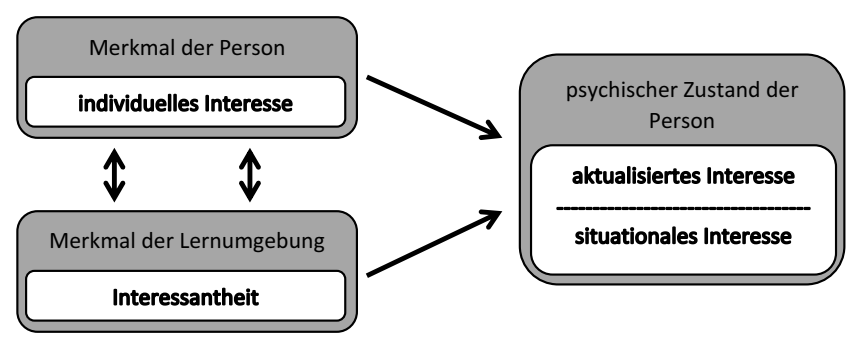

Abb. 1 Die relationale Struktur der Bedeutungsvarianten des Interessenkonstrukts. (Nach Krapp 1992c, S. 15) 
ner Tätigkeit differenziert (Hoffmann et al. 1998; Häußler 1987; Häußler und Hoffmann 2000), was wohl allgemein auf das naturwissenschaftliche Sachinteresse übertragen werden kann (Krapp 2010b; Blankenburg und Scheersoi 2018). Hemmer und Hemmer (2010a) unterscheiden - stärker geographiespezifisch - bei der Erfassung des Interesses zwischen dem Interesse an geographischen Themen, Regionen, Arbeitsweisen, wobei insbesondere letztere situationales Interesse hervorrufen können (Krapp und Ryan 2002).

Während domänenübergreifend bereits vielfältige Erkenntnisse zu themen- sowie situationsspezifischem Interesse vorliegen, so ist dieses bezogen auf den Themenkreis Klimawandel nur relativ wenig und kaum ausdifferenziert empirisch erforscht.

Den Schwerpunkt des Forschungsstandes stellt daher die allgemeine geographiedidaktische Interessenstudie von Hemmer und Hemmer (2010a, 2017) dar, in deren Rahmen querschnittlich im Abstand von zehn Jahren in Bayern sowie (in der Studie im Jahr 2015 zusätzlich in) Nordrhein-Westfalen jeweils 1600 (1995, 2005) bzw. 3400 (2015) Schüler*innen der Jahrgangsstufen 5-11 verschiedener Schulformen untersucht wurden. Während das aus inhaltlicher Sicht etwas undifferenziert zusammengesetzte Item „Treibhauseffekt und Ozonproblematik“ 1995 mit einem Mittelwert von $M=3,74$ (5-stufige Likert-Skala) noch Rangplatz 7 (von 50) erreichen und damit ein relativ interessantes Thema repräsentieren konnte, so erreichte das Item 2015 zwar einen positiven Interessenwert von $M=3,13$; allerdings nur noch Rang 45 von 57 (Wert nur für Gymnasien in NRW, $n=800$; Hemmer et al. 2019). Neben dem vergleichsweise geringen Schüler*inneninteresses ist weiterhin auffällig, dass das Item bei der 2015 ebenfalls durchgeführten Erhebung unter Lehrkräften mit $M=4,25$ auf Rang 9 rangiert (Hemmer und Hemmer 2017), was eine diesbezügliche Diskrepanz im Interesse der Schüler- und Lehrer*innen offenbart. Der Themenkreis Treibhauseffekt wurde auch in nicht spezifisch geographischen Interessenstudien untersucht - während im Rahmen der ROSE-Studie (The Relevance of Science Education), die naturwissenschaftsbezogene Lernvoraussetzungen auf internationaler Ebene erforscht hat, bei deutschen und österreichischen Schüler*innen ebenfalls ein Interessensverlust bezüglich der Thematik festzustellen war (Elster 2007), so stößt das Thema im Zuge der geowissenschaftlichen Interessenstudie „Forschungsdialog System Erde“ des IPN auf hohes Interesse (Rang 2 von 11; vgl. Hemmer et al. 2005). Auf internationaler Ebene untersuchten Carman et al. (2017) in den Vereinigten Staaten das Interesse von 121 zwölf- oder dreizehnjährigen Schüler*innen an den Auswirkungen des Klimawandels auf Wälder und konnten dabei zumindest ein moderat positives Interesse $(M=3,60$; 5-stufige LikertSkala) feststellen. Zwar ohne direkten Bezug zu Klima- wandel, aber mit gewissen Überschneidungen untersuchte Trend (2005) im Vereinigten Königreich bei 652 elf- sowie 12-jährigen Schüler*innen das Interesse an Geowissenschaften, wobei die beiden gebildeten Subskalen Weather sowie Water and Oceans zumindest Verwandtschaften zum Themenkreis Klimawandel aufweisen. Erstgenannte konnte mit $M=1,92$ (4-stufige Likert-Skala) Rangplatz drei, letztere mit $M=1,74$ Rangplatz fünf von sieben erreichen, wobei alle sieben gebildeten Subskalen im negativen Interessenbereich $(M<2,5)$ blieben. Ein allgemeiner Überblick über geographiedidaktische Interessensforschung soll an dieser Stelle mit Verweis auf Hemmer (2010) unterbleiben.

Durch die mit dem Thema Klimawandel verbundene besondere Relevanz potenzieller Handlungen (als mögliche Zieldimension von Geographieunterricht), soll an dieser Stelle ein knapper Verweis auf Konzepte der Handlungsmotivation erfolgen, die eine Verbindung zum Interessenkonstrukt aufweisen: Gemäß dem Rubikon-Modell der Handlungsregulation werden Handlungen von motivationalen sowie volitionalen Phasen determiniert (Heckhausen und Heckhausen 2010). Interesse bezeichnet dabei „eine besondere Motivationsform, die durch die Ausrichtung auf einen bestimmten Gegenstand charakterisiert ist" (Rheinberg 2010, S. 367; Krapp 1992b) und beeinflusst sowohl die prädezisionale als auch die postaktionale Phase der Handlungsregulation. In Eccles (2011) Erwartungs-mal-WertModell findet das Interesse als ein Bestimmungsfaktor der Wert-Komponente Eingang in das Motivationskonzept (Krapp und Ryan 2002). Die Theorie des geplanten Verhaltens (TPB) fasst die durch Einstellungen, subjektive Normen sowie wahrgenommene Verhaltenskontrolle bestimmten Verhaltensintentionen als motivationale Bedingungsfaktoren einer möglichen Handlung (Ajzen 1991) und berücksichtigt so das Interesse, Krapp (1992b) bezeichnet individuelle Interessen sogar als „Handlungsbereitschaften“ selbst und grenzt diese von den nach Bandura als Selbstwirksamkeitserwartungen bezeichneten Komponenten des Interesses ab, die stets von konkretisierten Problemstellungen bzw. Zielen abhängen (Krapp und Ryan 2002). Interesse stellt daher einen bedeutenden Faktor für potenzielles Handeln dar - wenn auch nicht im Sinne eines kausalen Automatismus, sondern vielmehr als einen Einflussfaktor eines äußerst komplexen/vielschichtigen Prozesses.

\section{Die Rolle von Kontexten in der Geographiedidaktik}

Bereits mehrere naturwissenschaftsdidaktische Untersuchungen konnten die Bedeutung der Kontexte, in die Themen im Unterricht eingebettet sind, für die Entwicklung des situationalen Interesses herausstellen (u. a. IPN-Interessenstudie Physik (Hoffmann et al. 1998) bzw. Geowissenschaften (Hemmer et al. 2005; Hlawatsch et al. 2005); ROSE (Sjøberg und Schreiner 2010); piko (Mikelskis-Seifert und 
Duit 2007), PLON (Kortland 2005); Salters (Bennett et al. 2005)). Unter einem Kontext wird zunächst das In Beziehung Setzen oder Einordnen eines Lerninhaltes in einen größeren Zusammenhang verstanden (Gilbert 2006; Fechner 2009; Parchmann und Kuhn 2018). Eine eindeutige und scharf abgrenzbare Definition des Kontextbegriffes - so das überhaupt möglich ist (Goodwin und Duranti 1992) - findet sich in der Literatur allerdings bisher nicht (van Oers 1998; Parchmann und Kuhn 2018). Während die neuere naturwissenschaftsdidaktische Forschung der PISA-Definition folgt und unter Kontexten ,life situations involving science and technology“ (OECD 2006, S. 25; Drechsel et al. 2011) versteht, so fassen andere Autoren darunter die konkreten Lernumgebungen, in denen ein Lernprozess stattfindet (Mikelskis-Seifert und Duit 2007; Finkelstein 2005). Ähnlich der Auffassung Muckenfuß (2004) werden Kontexte in diesem Beitrag als fachspezifische Betrachtungsweisen und Zugänge systematisiert, die verschiedene Blickwinkel auf ein fachliches Thema oder Themengebiet ermöglichen. Diese Systematisierung folgt dem Vorgehen prominenter naturwissenschaftsdidaktischer Interessenstudien (Hoffmann et al. 1998; Hemmer et al. 2005; Elster 2007; Bayrhuber et al. 2007) und ist nicht als Widerspruch zu obiger, auf lebensweltliche Problemsituationen ausgelegte Definition zu verstehen, sondern sieht diese als eine Komponente eines breiteren, an der spezifisch geographischen Fachsystematik orientierten Kontextverständnisses mit ähnlichen Zielsetzungen: Zum einen kann kontextorientierter Unterricht affektive Variablen wie das Interesse bzw. die Lernmotivation erhöhen und somit den Lernprozess unterstützen (Hemmer et al. 2005, 2011; van Vorst et al. 2015; Habig et al. 2018; OECD 2006) und zum anderen im Sinne der Ausbildung einer Scientific Literacy (OECD 2006; Nentwig et al. 2005) durch Variation der Kontextualisierung die Anwendung fachlicher Konzepte in alltäglichen bzw. außerunterrichtlichen Situationen und die Ausbildung einer inhaltlichen ,mental map“ fördern (Gilbert 2006, S. 959; OECD 2006; Habig et al. 2018; Bayrhuber et al. 2007; van Vorst et al. 2015; Parchmann und Kuhn 2018; Muckenfuß 2004; Mandl und Kopp 2005). Kontextbasierter Unterricht stellt daher auch einen wesentlichen Baustein für die in allen naturwissenschaftsdidaktischen Bildungsstandards verankerte Kompetenzorientierung dar (Weinert 2014; KMK 2005; DGfG 2017; Bayrhuber et al. 2007; Bayrhuber und Elster 2006).

Zur Erörterung des Forschungsstandes kontextbasierter Interessenstudien muss vorwiegend auf die naturwissenschaftsdidaktischen Nachbardisziplinen der Geographiedidaktik verwiesen werden. Bezogen auf das den PISA-Studien zugrundeliegende, primär lebensweltliche Verständnis von Kontexten stellten van Vorst et al. $(2015,2018)$ eine erste allgemeine Systematisierung zur Beurteilung des Einflusses verschiedener Kontextmerkmale auf die Inter- essantheit von Kontexten im Chemieunterricht auf. Einen guten Überblick über den, vorwiegend internationalen, Forschungsstand zu kontextbasiertem naturwissenschaftlichem Lernen in diesem Begriffsverständnis geben Nentwig und Waddington (2005). Für das diesem Artikel zugrunde liegende, stärker an der Fachsystematik orientierte Kontextverständnis stellt die prominente IPN-Interessenstudie Physik (Hoffmann et al. 1998) die initiale Basis dar, deren eher thematische Unterscheidung von Kontexten auch van Vorst et al. (2018) eine wesentliche Bedeutung beimisst. Diese über sechs Jahre (1984-1989) in sechs westdeutschen Bundesländern mit Längs- sowie Querschnittselementen durchgeführte Studie konnte zeigen, dass Kontexte verglichen mit dem physikalischen Fachgebiet bzw. der Tätigkeit einen wesentlich größeren Einfluss auf das Sachinteresse an Physik haben. Als am interessantesten wahrgenommen werden dabei grundsätzlich anwendungsbezogene, alltägliche, gesellschaftliche oder zum Staunen veranlassende Kontexte (Häußler und Hoffmann 1995). Die ROSE-Studie (Relevance Of Science Education) konnte für deutsche und österreichische Teilnehmer*innen ein besonderes Interesse an Kontexten ,in Zusammenhang mit Gesundheit, Fitness, Mystik und Spektakulärem“ (Elster 2007, S. 2) feststellen. Aus geographiedidaktischer Sicht besonders relevant ist die im Rahmen des Projektes „Forschungsdialog System Erde“ durchgeführte Studie zum Interesse an geowissenschaftlichen Themen (Hemmer et al. 2005; Hlawatsch et al. 2005), in der das Interesse an (den in Tab. 1 dargestellten) Themengebieten und Kontexten sowohl systematisch kombiniert als auch getrennt voneinander erfasst wurde. Bei den 333 Proband*innen der gymnasialen Oberstufe in Bayern, NRW und Schleswig-Holstein ergab sich erwartungsgemäß eine Abhängigkeit des Sachinteresses vom Kontext. Allgemein bestand ein verhältnismäßig hohes Interesse an den Kontexten Individuum, Gesellschaft und soziale Verantwor-

Tab. 1 Themengebiete und Kontexte des geowissenschaftlichen Teils von „Forschungsdialog System Erde“. (Nach Hemmer et al. 2005, S. 61)

\begin{tabular}{|c|c|c|c|}
\hline \multicolumn{2}{|c|}{ Themengebiete } & \multicolumn{2}{|c|}{ Kontexte } \\
\hline $\mathrm{T} 1$ & Teilsysteme der Erde & K1 & Individuell \\
\hline $\mathrm{T} 2$ & Kohlenstoffkreislauf & $\mathrm{K} 2$ & Gesellschaftlich \\
\hline $\mathrm{T} 3$ & $\begin{array}{l}\text { Gesteine und Minera- } \\
\text { le }\end{array}$ & K3 & $\begin{array}{l}\text { Normativ/soziale Verant- } \\
\text { wortung }\end{array}$ \\
\hline $\mathrm{T} 4$ & Fossile Rohstoffe & K4 & Systemisch \\
\hline T5 & Boden & K5 & Fachlich \\
\hline T6 & Gashydrate & K6 & Erdgeschichtlich \\
\hline $\mathrm{T} 7$ & Meer & K7 & Räumlich \\
\hline $\mathrm{T} 8$ & Trinkwasser & K8 & Wissenschaftsmethodisch \\
\hline T9 & Erdbeben & - & - \\
\hline $\mathrm{T} 10$ & Klimaänderungen & - & - \\
\hline $\mathrm{T} 11$ & $\begin{array}{l}\text { Veränderung der } \\
\text { Biodiversität }\end{array}$ & - & - \\
\hline
\end{tabular}


Abb. 2 Schematisches Raster zur Itemgenerierung

\begin{tabular}{|c|c|c|c|c|c|c|}
\hline Bereiche & individuell & $\begin{array}{l}\text { gesellschaft- } \\
\text { lich }\end{array}$ & lokal-regional & national & global & $\begin{array}{l}\text { wissen- } \\
\text { schaftsmetho- } \\
\text { disch }\end{array}$ \\
\hline \multicolumn{7}{|l|}{ Ursachen } \\
\hline Folgen & & & je Feld 2 Items & & & \\
\hline $\begin{array}{l}\text { Gegenmaß- } \\
\text { nahmen }\end{array}$ & & & & & & \\
\hline
\end{tabular}

tung, für Mädchen zusätzlich besonders an dem systemischen Kontext, bei Jungen am wissenschaftsmethodischen. Bezogen auf die Themengebiete wurde der Klimaänderung - wie bereits erwähnt - relativ hohes Interesse entgegengebracht, wofür insbesondere die Kontexte K1, K4, K5 sowie K7 ausschlaggebend waren. Hemmer et al. (2011) erfassten kontextbasiert das Interesse von 297 Schüler*innen der Jahrgangsstufe 6 (Haupt-, Realschule und Gymnasium) aus NRW an Naturkatastrophen. Auch hier stellte sich der individuelle Kontext als besonders interessant heraus, während dem wissenschaftsmethodischen Kontext leicht negatives Interesse entgegengebracht wurde.

\section{Methodik}

Ziel der vorliegenden Arbeit ist die Entwicklung und Validierung eines Instrumentes zur Messung des Interesses an den aus der Sicht der Schüler*innen hinter dem Themenkreis Klimawandel stehenden Strukturen. Da auf Basis des gegenwärtigen Forschungsstandes unklar ist, ob sich diese latenten Strukturen eher auf inhaltliche Facetten oder unterrichtliche Kontexte des Klimawandels beziehen und in welchem Maße diese in den Augen der Schüler*innen ausdifferenziert sind, werden Skalen entwickelt, die zum einen das Themengebiet aus geographisch-fachlicher bzw. geographiedidaktischer Sicht und zum anderen unterrichtliche Kontexte (systematisiert als geographische Fachsystematik bzw. Betrachtungsweisen) berücksichtigen. Aufgrund der besonderen fachlichen Nähe sollen die in Tab. 1 dargestellten Kontexte der IPN-Studie zum Interesse an Geowissenschaften (Hemmer et al. 2005) als Basis für eine klimawandelspezifische Kontextauswahl (s. Abschn. 3.1) dienen.

\section{Fachlich-inhaltliche Vorüberlegungen zur Itementwicklung}

Zur Entwicklung von Items, die das kontextbezogene Schüler*inneninteresse am Klimawandel inhaltsvalide erfassen, war es zunächst erforderlich, die Thematik sowohl aus geographisch-inhaltlicher Sicht als auch auf Ebene der geographiedidaktisch-unterrichtlichen Kontexte zu strukturieren. Die komplexe Thematik Klimawandel, die verschiedene geographische Sphären sowie deren Interaktion umfasst (Jacobeit 2007), sollte dabei in ihrer Prozesshaftigkeit durchaus ganzheitlich erfasst werden, keineswegs konnte es allerdings Ziel der Strukturierung sein, einzelne Details oder Spezifika explizit einzubeziehen. Die Thematik wird daher analog zu IPCC (2014) in die drei Bereiche Ursachen, Folgen und Gegenmaßnahmen strukturiert, wobei unter letzterem sowohl Anpassungs- als auch Vermeidungsstrategien gefasst werden. Auch wenn diese, auch in der Literatur häufig herangezogenen Bereiche (u. a. Rahmstorf und Schellnhuber 2019; Oelgeklaus 2012; Siegmund 2008) den Themenkreis Klimawandel nicht in seiner Gänze umfassen, zeichnen sie sich durch eine besondere gesellschaftliche und didaktische Relevanz aus. Zur Auswahl der relevanten Kontexte wurden die Vorschläge von Hemmer et al. (2005) (s. Tab. 1) herangezogen, die sich zum Teil auch mit den geographiedidaktischen Basiskonzepten (Fögele 2018) decken. Die der Itementwicklung letztendlich zugrunde gelegten Kontexte sind in Abb. 2 ersichtlich.

Um ein möglichst komprimiertes und so auch in der Unterrichtspraxis einsetzbares Messinstrument zu erhalten, musste - auch unter Inkaufnahme eines gewissen Informationsverlustes - eine themenbezogene Auswahl bzw. Priorisierung der Kontexte vorgenommen werden. Das Kontextpaar individuell - gesellschaftlich spielt insbesondere dahingehend eine Rolle, weil es sich beim Klimawandel um ein potenziell handlungs- sowie zukunftsrelevantes Thema handelt, das von deutschen Schüler*innen allerdings tendenziell als „Fernthema“ wahrgenommen wird (Schuler 2009; Höhnle 2014). Offen ist, ob eher an der individuellen oder gesellschaftlichen Dimension dieses Themenkreises Interesse besteht. Auf die inhaltlich für die Thematik zwar äuBerst relevanten Kontexte normativ/soziale Verantwortung, (nicht) systemisch sowie erdgeschichtlich/zeitlich wurde im Vergleich zu Hemmer et al. (2005) zugunsten einer differenzierten räumlichen Kontextebene verzichtet. Raum stellt die zentrale Kategorie der Geographie dar und wird u.a. ,auf unterschiedlichen Maßstabsebenen“" (DGfG 2017, S. 6) analysiert. Da die damit einhergehende geographiespezifische Analysestruktur für den Klimawandel durch die räumliche Entkopplung der einzelnen Prozesskomponenten eine herausgehobene Stellung einnimmt, wurde der räumliche Kontext gemäß dem Basiskonzept Maßstabsebenen(wechsel) in die Kategorien regional, national und global ausdifferenziert (Chang und Pascua 2017; Fögele 2018). Der weiterhin berücksichtigte wissenschaftsmethodische Kontext ist vor allem dahingehend relevant, dass dieser typisch geogra- 
phische Arbeitsweisen erfasst sowie den Klimawandel im Licht stetiger wissenschaftlicher Weiterentwicklung (IPCC 2014) betrachtet, durch die Jugendliche permanent mit einer Fülle an mehr oder weniger seriösen Neuinformationen konfrontiert werden. Auch mit Blick auf den aktuellen Forschungsstand scheint die Untersuchung dieses Kontextes besonders relevant, so liegen hier - z. B. in Verbindung mit dem Thema Erdbeben/Naturkatastrophen - ambivalente Befunde bezüglich des Interesses vor (Hemmer et al. 2005, 2011).

\section{Itemanalyse und Skalenentwicklung /-bildung}

Um die Komplexität der Studie zu reduzieren und ein handhabbares Messinstrument im Sinne einer hohen ökologischen Validität (Döring und Bortz 2016) zu generieren, wird das Interesse in Anlehnung an die IPN-Studien grundsätzlich als globales Konstrukt gemessen. Aufgrund der besonderen Bedeutung der Kontexte für die Interessensgenese (Häußler 1987; Häußler und Hoffmann 1995; Hemmer und Hemmer 2010b) sind alle Items allerdings so strukturiert, dass sie neben dem Interesse an einer Inhaltskomponente des Klimawandels stets auch das Interesse an einem unterrichtlichen Kontext erfassen. Die inhaltlichen Komponenten sowie Kontexte wurden daher systematisch kombiniert und jedes Feld (s. Abb. 2) mit zwei Items besetzt. Es ergab sich ein Fragebogen mit 36 Items, die zur Minimierung unerwünschter Reihenfolgeeffekte (Döring und Bortz 2016) zufällig angeordnet wurden. Die Fragebogenerhebung mit einer Dauer von zehn Minuten fand im Rahmen einer Informationsveranstaltung für ein universitäres Kooperationsprojekt mit Schulen zwischen Januar und März 2020 an einer für explorative Faktoranalysen zumindest ausreichend großen Stichprobe (Bühner 2011) von 144 Schüler*innen der Jahrgangsstufen 10 und 11 zehn bayerischer Gymnasien vor Behandlung des Themas Klimawandel statt. Um eine Antworttendenz zur Mitte zu vermeiden, erfolgte die Bewertung der Aussagen abweichend zu Hemmer et al. (2005) anhand einer sechsstufigen likertartigen Skala (Döring und Bortz 2016) mit den Extremen interessiert mich gar nicht (1) bzw. interessiert mich sehr (6). Da die Erhebung ausschließlich der Aufdeckung der Faktorenstruktur diente, wurden keine biographischen Kontrollvariablen erhoben. Die Auswertung der Daten erfolgte mit IBM SPSS Statistics 26. Eine Analyse der fehlenden Werte brachte dabei nur bei vier Items Datenlücken hervor (insgesamt $0,14 \%$ fehlende Werte). Little's MCAR-Test $\left(\chi^{2}=257,32\right.$; df $=174 ; p<0,001)$ konnte das völlig zufällige Fehlen der Werte nicht nachweisen, es kann allerdings mangels jedweder erkennbarer Systematik der Datenlücken von Missing At Random ausgegangen werden (Lüdtke et al. 2007). Die fehlenden Werte wurden daher mittels des sowohl für Faktoren- als auch Reliabilitätsanalysen grundsätzlich gut geeigneten EM-Algorithmus imputiert, da dieser die Verletzung der Normalverteilungsannahme bei nur sehr geringer Datenausfallquote gut toleriert und derartige Verfahren trotz fehlender Berücksichtigung von Residualvarianzen (Graham 2009) zu ,valideren Ergebnissen führen als die klassischen Ansätze“ (Lüdtke et al. 2007, S. 116) der Datenimputation.

Da explizit kein zu prüfendes Theoriemodell vorliegt, sondern die Items lediglich auf Basis einer theoriegeleiteten Matrix konstruiert wurden, wurde zur Aufdeckung der empirisch beobachteten Faktorenstruktur eine explorative Faktorenanalyse durchgeführt. Die grundsätzliche Eignung dieser Analyseform wurde mittels des Kaiser-Meyer-OlkinKoeffizienten (KMO), des Barlett-Tests sowie der AntiImage-Kovarianz-Matrix überprüft. Sowohl $\mathrm{KMO}=0,924$ (marvelous), Barlett-Test $\left(\chi^{2}=3617,58 ; \mathrm{df}=630 ; p<0,001\right)$ als auch die Anti-Image-Matrix sprechen nicht gegen die Eignung der Korrelationsmatrix für eine Faktorenanalyse (Bühner 2011; Backhaus et al. 2016).

Als Extraktionsmethode wurde die gebräuchliche Maximum-Likelihood-Analyse (ML) gewählt, da latente Dimensionen hinter der Variablenstruktur vermutet werden; zur Bestimmung der Faktorenzahl wurde allerdings zunächst eine Hauptkomponentenanalyse durchgeführt (Bühner 2011). Während der optische Scree-Test die Extraktion nur eines Faktors und die Parallelanalyse nach Horn zwei Faktoren vorschlägt, empfiehlt der Minimum-Average-Partial-Test $(M A P)$, der den beiden erstgenannten Analysen unter den gegebenen Bedingungen vorzuziehen ist (Bühner 2011), die Extraktion von fünf Faktoren. Gemäß dem KaiserGuttmann-Kriterium (Eigenwert >1) sind 7 Faktoren $\mathrm{zu}$ extrahieren, aufgrund der hohen Reliabilität der Gesamtskala $(\alpha=0,960)$ kann dieses Kriterium auch bedenkenlos herangezogen werden (Bühner 2011). Der im Zuge der späteren ML-Analyse durchgeführte Chi $^{2}$-Test auf Güte der Anpassung zeigt allerdings keine perfekte Passung mit den Daten $\left(\chi^{2}=506,94 ; \mathrm{df}=399 ; p<0,001\right.$; Schendera 2010). Mit einem RMSEA $=0,04$ erreicht das Modell allerdings einen ausreichenden approximativen Fit (Moschopoulos 1983; Browne und Cudeck 1993). Als Rotationmethode wurde aufgrund der zum Teil hohen Interfaktorenkorrelation die oblique Promax-Rotation $(\kappa=4)$ gewählt (Bühner 2011).

\section{Ergebnisse}

Es ergaben sich fünf sinnvoll interpretierbare Faktoren (s. Tab. 2), die anfänglich 57,73\% der Gesamtvarianz des Modells aufklären konnten. Die Korrelation der extrahierten Faktoren ist insbesondere zwischen den ersten beiden Faktoren mit $r=0,730$ relativ hoch, die eigenständige Interpretierbarkeit eines Faktors gilt analog zu PISA 
Tab. 2 Mustermatrix der Promax-rotierten Maximum-Likelihood-Analyse sowie Reliabilitätskennzahlen und Mittelwerte der identifizierten Skalen

\begin{tabular}{|c|c|c|c|c|c|c|}
\hline Skala & Itemwortlaut & 1 & 2 & 3 & 4 & 5 \\
\hline \multirow[t]{7}{*}{ Handlung } & $\begin{array}{l}\text { (1) Welche Möglichkeiten ich persönlich habe, um den } \\
\text { Klimawandel zu begrenzen ... }\end{array}$ & 0,965 & - & - & - & - \\
\hline & $\begin{array}{l}\text { (2) Wie ich persönlich zum Voranschreiten des Klimawan- } \\
\text { dels beitrage ... }\end{array}$ & 0,865 & - & - & - & - \\
\hline & $\begin{array}{l}\text { (3) Welche Möglichkeiten ich im Alltag habe, mich an die } \\
\text { Veränderungen durch den Klimawandel anzupassen ... }\end{array}$ & $\mathbf{0 , 8 1 8}$ & - & - & - & - \\
\hline & $\begin{array}{l}\text { (4) Inwieweit meine alltäglichen Handlungen den Klima- } \\
\text { wandel mitverursachen ... }\end{array}$ & 0,809 & - & - & - & - \\
\hline & $\begin{array}{l}\text { (5) Wie der Lebensstil in unserer Gesellschaft den Klima- } \\
\text { wandel verstärkt ... }\end{array}$ & 0,673 & - & - & - & - \\
\hline & $\begin{array}{l}\text { (6) Was in Deutschland getan werden kann, um den } \mathrm{CO}_{2-} \\
\text { Ausstoß zu reduzieren ... }\end{array}$ & 0,648 & - & - & - & - \\
\hline & $\begin{array}{l}\text { (7) Welche Maßnahmen in Deutschland ergriffen werden } \\
\text { können, um mit dem Klimawandel umzugehen ... }\end{array}$ & 0,579 & - & - & - & - \\
\hline \multirow[t]{6}{*}{$\begin{array}{l}\text { Räumliche } \\
\text { Nähe }\end{array}$} & $\begin{array}{l}\text { (8) Wie mein Heimatort vom Klimawandel betroffen sein } \\
\text { wird ... }\end{array}$ & - & $1, \mathbf{0 8 4}^{\mathrm{a}}$ & - & - & - \\
\hline & $\begin{array}{l}\text { (9) Inwieweit Deutschland von den Folgen des Klimawan- } \\
\text { dels betroffen ist ... }\end{array}$ & - & 0,773 & - & - & - \\
\hline & $\begin{array}{l}\text { (10) Durch welche Maßnahmen in meinem Heimatdorf } \\
\text { zum Klimaschutz beigetragen werden kann ... }\end{array}$ & - & $\mathbf{0 , 7 3 3}$ & - & - & - \\
\hline & $\begin{array}{l}\text { (11) Durch welche Prozesse in meiner Heimatregion der } \\
\text { Klimawandel verstärkt wurde ... }\end{array}$ & - & 0,637 & - & - & - \\
\hline & $\begin{array}{l}\text { (12) Wie sich mein Wohnort durch Veränderungen vor } \\
\text { dem Klimawandel schützen kann ... }\end{array}$ & 0,308 & 0,572 & - & - & - \\
\hline & $\begin{array}{l}\text { (13) Inwieweit der Klimawandel auch dort, wo ich wohne, } \\
\text { hervorgerufen wird ... }\end{array}$ & 0,352 & 0,565 & - & - & - \\
\hline \multirow{3}{*}{$\begin{array}{l}\text { Globale } \\
\text { Ursa- } \\
\text { chen }\end{array}$} & $\begin{array}{l}\text { (14) Was global gesehen am stärksten zum Klimawandel } \\
\text { beiträgt ... }\end{array}$ & - & - & $\mathbf{0 , 8 5 4}$ & - & - \\
\hline & $\begin{array}{l}\text { (15) Welche Länder weltweit besonders für den Klima- } \\
\text { wandel verantwortlich sind ... }\end{array}$ & - & - & 0,722 & - & - \\
\hline & $\begin{array}{l}\text { (16) Welche Bereiche (z. B. Verkehr, Landwirtschaft, In- } \\
\text { dustrie ...) in Deutschland den größten Beitrag zum Klima- } \\
\text { wandel leisten }\end{array}$ & - & - & $\mathbf{0 , 5 5 0}$ & - & - \\
\hline \multirow[t]{4}{*}{$\begin{array}{l}\text { Messung/ } \\
\text { Methodik }\end{array}$} & $\begin{array}{l}\text { (17) Wie die Kosten für Schutzmaßnahmen vor dem Kli- } \\
\text { mawandel errechnet werden ... }\end{array}$ & - & - & - & $\mathbf{0 , 8 3 4}$ & - \\
\hline & $\begin{array}{l}\text { (18) Wie nachgewiesen werden kann, dass Treibhausgase } \\
\text { die Ursachen für den Klimawandel sind ... }\end{array}$ & - & - & - & 0,640 & - \\
\hline & $\begin{array}{l}\text { (19) Mit welchen Methoden der } \mathrm{CO}_{2} \text {-Ausstoß gemessen } \\
\text { wird ... }\end{array}$ & - & - & - & 0,560 & - \\
\hline & $\begin{array}{l}\text { (20) Wie die Folgen des Klimawandels gemessen werden } \\
\text { können ... }\end{array}$ & - & - & - & $\mathbf{0 , 5 5 8}$ & - \\
\hline
\end{tabular}

bei Interfaktorkorrelationen bis $r=0,85$ als gegeben (Walter und Taskinen 2008). Substanzielle Doppelladungen $(>0,30)$ kamen nur in wenigen Fällen vor und konnten sowohl auf Basis inhaltlicher Überlegungen als auch des FürntrattKriteriums (Fürntratt 1969) stets eindeutig einem Faktor zugeordnet werden, eine Einfachstruktur liegt demnach vor (Döring und Bortz 2016). Variablen mit Faktorladungen $<0,55$ sowie Faktoren mit weniger als drei Variablen wurden gemäß Schenderas (2010) Empfehlung bei der Skalenbildung nicht berücksichtigt Die Split-Half-Reliabilität der Skalen mit einem Spearman-Brown-Koeffizienten von 0,804-0,923 sowie die interne Konsistenz mit Cronbachs-Alpha von 0,821-0,925 liegen mit Ausnahme einer Skala $(\alpha=0,748 ; r=0,766)$ für alle Skalen im mittleren bis hohen Bereich. Die Itemschwierigkeiten liegen mit einem Mittelwert des Schwierigkeitsindex von 0,68 zwar eher im höheren (d.h. leichteren) Bereich, die von Döring und Bortz (2016) vorgeschlagenen Grenzwerte von 0,20 bzw. 0,80 werden allerdings von keinem Item unter- bzw. überschritten.

Die erste Skala (Handlung; Items 01-07) beschreibt den klimawandelbezogenen Handlungskontext sowohl auf in- 
Tab. 2 (Fortsetzung)

\begin{tabular}{|c|c|c|c|c|c|c|}
\hline Skala & Itemwortlaut & 1 & 2 & 3 & 4 & 5 \\
\hline \multirow[t]{3}{*}{$\begin{array}{l}\text { Globale } \\
\text { Folgen }\end{array}$} & $\begin{array}{l}\text { (21) Welche Folgen der Klimawandel für weit entfernte } \\
\text { Länder mit sich bringt ... }\end{array}$ & - & - & - & - & $\overline{0,745}$ \\
\hline & $\begin{array}{l}\text { (22) Welche Folgen der Klimawandel für unsere Gesell- } \\
\text { schaft hat ... }\end{array}$ & - & - & - & - & 0,671 \\
\hline & $\begin{array}{l}\text { (23) Wo auf der Erde die Folgen des Klimawandels beson- } \\
\text { ders stark spürbar sind ... }\end{array}$ & - & - & - & - & $\mathbf{0 , 5 9 7}$ \\
\hline - & Rotierte Eigenwerte $^{c}$ & 8,53 & 6,42 & 5,33 & 4,22 & 5,29 \\
\hline- & Aufgeklärte Varianz (in \%) & 41,27 & 7,38 & 5,10 & 4,18 & 3,31 \\
\hline- & Split-Half-Reliabilität & 0,923 & 0,827 & 0,804 & 0,766 & 0,820 \\
\hline- & Cronbachs- $\alpha$ & 0,925 & 0,888 & 0,824 & 0,748 & 0,821 \\
\hline- & $M(S D)$ Skala & $\begin{array}{l}4,71 \\
(1,15)\end{array}$ & $\begin{array}{l}4,30 \\
(1,13)\end{array}$ & $\begin{array}{l}4,61 \\
(1,16)\end{array}$ & $\begin{array}{l}3,69 \\
(1,06)\end{array}$ & $\begin{array}{l}4,47 \\
(1,13)\end{array}$ \\
\hline
\end{tabular}

Items im Originalwortlaut bei sechsstufiger likertartiger Skala mit Ausprägungen ,... interessiert mich gar nicht“ (1) bis ,,... interessiert mich sehr“ (6); Ladungen $<0,30$ werden nicht dargestellt

${ }^{a}$ Ladungen $>1$ sind bei obliquen Rotationsmethoden möglich, da es sich um Regressionskoeffizienten, nicht um Korrelationen handelt (Jöreskog 1999)

${ }^{\mathrm{b}}$ Bei dieser Variable liegt eine Nebenladung auf einen weiteren, nicht interpretierbaren Faktor $(\lambda=0,474)$ vor

${ }^{\mathrm{c}}$ Die Berechnung erfolgte unter Ausschluss der (in dieser Tabelle nicht angeführten) aus den Skalen jeweils eliminierten Variablen

${ }^{\mathrm{d}}$ Vor Rotation (Bühner 2011)

dividueller als auch gesellschaftlicher Ebene. Aus inhaltlichen Überlegungen musste aus der Skala ein Item (Wie sich der Klimawandel auf mich persönlich auswirkt ...) entfernt werden. Items 08-13 bilden die Skala räumliche Nähe, die einen (sozial-)räumlich begrenzten unterrichtlichen Kontext des Klimawandels darstellt, bezogen auf die inhaltlichen Bereiche des Klimawandels allerdings durchaus ausdifferenziert ist. In Abgrenzung dazu werden die eher globalen Ursachen des Klimawandels in der dritten Skala (Items 14-16) dargestellt. Die vierte Skala (Items 17-20; Messung/ Methodik) repräsentiert den forschungsmethodischen Kontext des Klimawandels, ohne dabei die eigene, handelndaktive Anwendung naturwissenschaftlicher Arbeitsweisen durch die Schüler*innen zu fordern. Die fünfte Skala (Items 21-23) beleuchtet als Pendant zur Ursachen-Skala die globalen Folgen des Klimawandels.

\section{Diskussion}

Ziel der vorgestellten Studie war die Konstruktion und Faktorenanalyse eines Fragebogens zum Interesse von Schüler*innen am Thema Klimawandel bzw. der aus Sicht der Schüler*innen hinter dem Klimawandel stehenden latenten Strukturen. Diese an den Perspektiven der Schüler*innen orientierte Ausdifferenzierung stellt einen für die Geographiedidaktik neuen Befund dar. Auf Basis des bisherigen Forschungsstandes konnte keine hypothesenprüfende konfirmatorische Faktorenanalyse, sondern zunächst eine hypothesengenerierende explorative Faktorenanalyse durchgeführt werden. Einschränkend muss allerdings erwähnt werden, dass die Ausrichtung an den
Schüler*innenperspektiven zwangsläufig mit einer Lösung von den fachlich-inhaltlichen Strukturen einhergeht, sodass in Kauf genommen werden muss, dass Teile der Fachsystematik nicht abgebildet werden. Dies zeigt sich bereits bei der selektiven Auswahl der unterrichtlichen Kontexte im Zuge der Entwicklung des Messinstrumentes. Durch die anschließende Itemreduktion im Zuge der Skalenbildung geht zwar ein weiterer Informationsverlust einher, allerdings entstand so eine Skala mit nur 23 Items, die sich aufgrund ihrer zeitökonomischen Handhabbarkeit insbesondere auch für den Einsatz in der Unterrichtspraxis eignet. Zur ersten Erprobung wurde der Fragebogen zunächst nur am Gymnasium an einer eher kleinen Stichprobe eingesetzt und keine zusätzlichen Kontrollvariablen erhoben, was die Verallgemeinerbarkeit der gefundenen Strukturen einschränkt und ihren explorativen Charakter aufzeigt. Daher scheint es angezeigt, die gefundenen Skalen, die in einer weiteren Studie als Ausgangsmodell herangezogen werden können, einem hypothesenprüfenden Verfahren (z.B. einem Strukturgleichungsmodell) zu unterziehen. Im Zuge der Itemanalyse ergaben sich sowohl kontextuelle (Handlung, räumliche Nähe, Messung/Methodik) als auch inhaltlichthematische (globale Ursachen, globale Folgen) Skalen. Eine tiefergehende Betrachtung der einzelnen Skalen zeigt aber, dass die unterrichtlichen Kontexte im Sinne von fachspezifischen Zugängen für die Struktur der Schüler*innen leitend sind: Obwohl sich für sowohl Ursachen als auch Folgen des Klimawandels eigene Skalen bilden, finden sich besonders in den Skalen Handlung sowie räumliche Nähe alle Items zu Ursachen oder Folgen, die einen Handlungs- bzw. Nahraumbezug (im Sinne eines sowohl objektiv-realistischen als auch subjektiv-konstruktivistischen 
Nahraum- bzw. Heimatverständnisses) aufweisen. Hierbei fällt in beiden Skalen die starke Konzentration von Items mit individuellem und etwas abgeschwächt mit nationalgesellschaftlichem Bezug auf. Dies signalisiert, dass der Klimawandel durchaus als „Handlungsthema“ und bezogen auf klimarelevante Handlungen durchaus eine individuelle Verantwortung der Proband*innen wahrgenommen wird. Besonders relevant erscheint dieser vor dem Hintergrund, dass kontextbezogene Interessenforschung bisher lebensweltliche Kontexte sowohl individueller als auch gesellschaftlicher Ausprägung als besonders interessenförderlich herausstellen konnte (u.a. Häußler und Hoffmann 1995; Hemmer et al. 2005; Hemmer und Hemmer 2010b). Dies kann analog daher auch für die Kontexte Handlung sowie räumliche Nähe erwartet werden, in denen sich Items dieser beiden Bereiche konzentrieren. Dieser lebensweltliche Kontext äußert sich bezogen auf den Klimawandel in dichotomer Ausprägung - zum einen bezogen auf (sozial)räumliche Nähe und zum anderen auf einer handlungsbezogenen Ebene. Die besondere Bedeutung dieser beiden Kontexte als Determinanten des Interesses wird auch durch deren hohe Varianzaufklärung verdeutlicht (kumuliert $49 \%$ bei insgesamt $61 \%$ aufgeklärter Varianz). Die Skalen globale Ursachen sowie globale Folgen sind folgerichtig schwerpunktmäßig mit Variablen des globalen Maßstabs besetzt und untermauern, dass unterrichtliche Konstellationen mit handlungs- oder nahraumspezifischen Bezügen von Schüler*innen im Licht dieser Kontexte wahrgenommen werden und sich erst darüber hinaus auf die Inhaltsbereiche des Klimawandels ausdifferenzieren. Während zur Messung des Interesses an der globalen Maßstabsebene des Klimawandels die beiden Kontexte globale Ursachen und globale Folgen gemeinsam herangezogen werden können, ist eine zielgenaue Erfassung des Interesses an z.B. allgemeinen Ursachen oder Folgen wohl nur eingeschränkt möglich, da die regionale Komponente als das Interesse determinierender Faktor nicht gänzlich eliminiert werden kann. Obwohl die vorliegende Stichprobe valide inhaltliche Interpretationen des Interesses nur begrenzt zulässt, liegt mit Blick auf die Mittelwerte der Skala Messung/Methodik zumindest die Vermutung nahe, dass dieser Kontext ein vergleichsweise niedriges Interesse hervorruft, weshalb bei methodenspezifischem Klimawandelunterricht didaktisch besonders umsichtig vorgegangen werden sollte. Der bisherigen Argumentation folgend wäre es durchaus vertretbar, die Skalen globale Ursachen sowie Folgen aus dem Messinstrument zu entfernen; um den Informationsverlust allerdings möglichst gering zu halten, werden diese vorerst beibehalten. Zu überlegen ist weiterhin, den Fragebogen um die für den Themenkreis Klimawandel äußerst relevante politische Dimension zu erweitern, da entsprechende Items aus messmethodischen Gründen gänzlich aus dem Fragebogen entnommen werden mussten. Für die Skala räumliche Nähe sollte in Form einer qualitativen Interviewstudie geprüft werden, ob Heimat (in Abgrenzung von z.B. der Formulierung Wohnort) von den Schüler*innen tatsächlich individuell interpretiert wird oder - entgegen der Intention der Autoren - in einem objektiv-realistischen Sinne ausgelegt wird. Da der Fragebogen unabhängig von Klimawandelunterricht eingesetzt wurde, liegt nahe, dass dieser schwerpunkmäßig das individuelle Interesse bzw. die trait-Komponente des Interesses misst. Dies stellt im Sinne einer intrinsischen Motivation oder gar Handlungsbereitschaft eine wesentliche Grundlage für klimabezogenes Handeln dar und deckt sich mit der besonderen Bedeutung der Skala Handlung in dieser Studie.

Die entwickelten Skalen schaffen die Voraussetzungen, das Interesse am zunehmend aktueller werdenden Thema Klimawandel zu erfassen sowie die interessenbezogene Wirksamkeit unterrichtlicher Interventionen zu überprüfen, wodurch eine wertvolle Voraussetzung für künftigen Klimawandelunterricht und fachdidaktische Forschung geschaffen wird.

Förderung Dieses Forschungsprojekt wurde im Rahmen des Bayerischen Netzwerks für Klimaforschung (bayklif) vom Bayerischen Staatsministerium für Wissenschaft und Kunst gefördert.

Funding Open Access funding enabled and organized by Projekt DEAL.

Open Access Dieser Artikel wird unter der Creative Commons Namensnennung 4.0 International Lizenz veröffentlicht, welche die Nutzung, Vervielfältigung, Bearbeitung, Verbreitung und Wiedergabe in jeglichem Medium und Format erlaubt, sofern Sie den/die ursprünglichen Autor(en) und die Quelle ordnungsgemäß nennen, einen Link zur Creative Commons Lizenz beifügen und angeben, ob Änderungen vorgenommen wurden.

Die in diesem Artikel enthaltenen Bilder und sonstiges Drittmaterial unterliegen ebenfalls der genannten Creative Commons Lizenz, sofern sich aus der Abbildungslegende nichts anderes ergibt. Sofern das betreffende Material nicht unter der genannten Creative Commons Lizenz steht und die betreffende Handlung nicht nach gesetzlichen Vorschriften erlaubt ist, ist für die oben aufgeführten Weiterverwendungen des Materials die Einwilligung des jeweiligen Rechteinhabers einzuholen.

Weitere Details zur Lizenz entnehmen Sie bitte der Lizenzinformation auf http://creativecommons.org/licenses/by/4.0/deed.de.

\section{Literatur}

Ajzen, I. (1991). The theory of planned behavior. Organizational Behavior and Human Decision Processes, 50(2), 179-211.

Backhaus, K., Erichson, B., Plinke, W., \& Weiber, R. (2016). Multivariate Analysemethoden. Eine anwendungsorientierte Einführung. Berlin, Heidelberg: Springer Gabler.

Bayrhuber, H., \& Elster, D. (2006). Outcome orientation of biology teaching. Kiel. https://www.researchgate.net/profile/Doris_ Elster/publication/269708422_Outcome_Orientation_in_Biology_ Education/links/54a422450cf256bf8bb31e0b.pdf. Zugegriffen: 12. Febr. 2021.

Bayrhuber, H., Bögeholz, S., Elster, D., Hammann, M., Hössle, C., Lücken, M., et al. (2007). Biologie im Kontext. Ein Programm 
zur Kompetenzförderung durch Kontextorientierung im Biologieunterricht und zur Unterstützung von Lehrerprofessionalisierung. Der mathematische und naturwissenschaftliche Unterricht (MNU), 60(5), 282-286.

Bennett, J., Holman, J., Lubben, F., Nicolson, P., \& Otter, C. (2005). Science in context: the salters approach. In P. Nentwig \& D.J. Waddington (Hrsg.), Making it relevant. Context based learning of science (S. 121-153). Münster: Waxmann.

Blankenburg, J., \& Scheersoi, A. (2018). Interesse und Interessenentwicklung. In D. Krüger, I. Parchmann \& H. Schecker (Hrsg.), Theorien in der naturwissenschaftsdidaktischen Forschung (S. 245-259). Berlin, Heidelberg: Springer.

Borsdorf, A. (2019). Geographisch denken und wissenschaftlich arbeiten

Browne, M. A., \& Cudeck, R. (1993). Alternative ways of assessing model fit. In K. A. Bollen \& J. S. Long (Hrsg.), Testing structural equation models (S. 136-162). Newbury Park: SAGE.

Bühner, M. (2011). Einführung in die Test- und Fragebogenkonstruktion. München: Pearson Studium.

Carman, J., Zint, M., \& Ibanez, I. (2017). Assesing student interest and desire to learn more about climate change effects on forests in middle school: an intervention-based path model. Electronic Journal of Science of Education, 21(5), 14-35.

Chang, C.-H., \& Pascua, L. (2017). Geographical thinking and its role in climate change education: the case of Singapore. In C. Brooks, G. Butt \& M. Fargher (Hrsg.), The power of geographical thinking (S. 91-102). Cham: Springer.

Csikszentmihalyi, M. (1999). If we are so rich, why aren't we happy? American Psychologist, 54(10), 821-827.

Deutsche Gesellschaft für Geographie (DGfG) (2017). Bildungsstandards im Fach Geographie für den Mittleren Schulabschluss mit Aufgabenbeispielen. Bonn: DGfG.

Döring, N., \& Bortz, J. (2016). Forschungsmethoden und Evaluation in den Sozial- und Humanwissenschaften. Berlin, Heidelberg: Springer.

Drechsel, B., Carstensen, C., \& Prenzel, M. (2011). The role of content and context in PISA interest scales: a study of the embedded interest items in the PISA 2006 science assessment. International Journal of Science Education, 33(1), 73-95.

Eccles, J. (2011). Gendered educational and occupational choices: applying the Eccles et al. model of achievement-related choices. International Journal of Behavioral Development, 35(3), 195-201.

Elster, D. (2007). In welchen Kontexten sind naturwissenschaftliche Inhalte für Jugendliche interessant? Ergebnisse der ROSE-Erhebung in Österreich und Deutschland. Plus Lucis, 15(3), 2-8.

Endlicher, W., \& Gerstengarbe, F.-W. (Hrsg.). (2007). Der Klimawandel. Einblicke, Rückblicke und Ausblicke. Potsdam: Potsdam-Institut für Klimafolgenforschung e.V.

Fechner, S. (2009). Effects of context oriented learning on student interest and achievement in chemistry education. Berlin: Logos.

Finkelstein, N. (2005). Learning physics in context: a study of student learning about electricity and magnetism. International Journal of Science Education, 27(10), 1187-1209.

Fögele, J. (2018). Lehrertypen im Umgang mit geographischen Basiskonzepten. Rekonstruktion professioneller Überzeugungen von Geographielehrkräften. Zeitschrift für Geographiedidaktik (ZGD), 46(1), 3-32.

Fürntratt, E. (1969). Zur Bestimmung der Anzahl interpretierbarer gemeinsamer Faktoren in Faktorenanalysen psychologischer Daten. Diagnostica, 15, 62-75.

Gebhard, U., Höttecke, D., \& Rehm, M. (2017). Pädagogik der Naturwissenschaften. Ein Studienbuch. Wiesbaden: Springer VS.

Gilbert, J. K. (2006). On the nature of "context" in chemical education. International Journey of Science Education, 28(9), 957-976.

Goodwin, C., \& Duranti, A. (1992). Rethinking context: an introduction. In A. Duranti \& C. Goodwin (Hrsg.), Rethinking context. Language as an interactive phenomenon (S. 1-42). Cambridge, New York, Melbourne: Cambridge University Press.
Graham, J. W. (2009). Missing data analysis: making it work in the real world. Annual review of psychology, 60, 549-576.

Habig, S., van Vorst, H., \& Sumfleth, E. (2018). Merkmale kontextualisierter Lernaufgaben und ihre Wirkung auf das situationale Interesse und die Lernleistung von Schülerinnen und Schülern. ZfDN, 24(1), 99-114

Hattie, J. (2012). Visible learning for teachers. Maximizing impact on learning. London, New York: Routledge Taylor \& Francis Group.

Häußler, P. (1987). Measuring students' interest in physics-design and results of a cross-sectional study in the Federal Republic of Germany. International Journal of Science Education, 9(1), 79-92.

Häußler, P., \& Hoffmann, L. (1995). Physikunterricht - an den Interessen von Mädchen und Jungen orientiert. Unterrichtswissenschaft, 23(2), 107-126.

Häußler, P., \& Hoffmann, L. (2000). A curricular frame for physics education: development, comparison with students' interests, and impact on students' achievement and self-concept. Science Education, 84(6), 689-705.

Häußler, P., Hoffmann, L., Langeheine, R., Rost, J., \& Sievers, K. (1996). Qualitative Unterschiede im Interesse an Physik und Konsequenzen für den Physikunterricht. Zeitschrift für Didaktik der Naturwissenschaften (ZfDN), 2(3), 57-69.

Heckhausen, J., \& Heckhausen, H. (2010). Motivation und Handeln: Einführung und Überblick. In J. Heckhausen \& H. Heckhausen (Hrsg.), Motivation und Handeln (S. 1-9). Berlin, Heidelberg: Springer.

Helmke, A. (2017). Unterrichtsqualität und Lehrerprofessionalität. Diagnose, Evaluation und Verbesserung des Unterrichts. SeelzeVelber: Klett.

Helmke, A., \& Schrader, F.-W. (2010). Determinanten der Schulleistung. In D. H. Rost (Hrsg.), Handwörterbuch Pädagogische Psychologie (S. 90-102). Weinheim: Beltz.

Helmke, A., \& Weinert, F.E. (1997). Bedingungsfaktoren schulischer Leistung. In F.E. Weinert, N. Birbaumer \& C.F. Graumann (Hrsg.), Psychologie des Unterrichts und der Schule (S. 71-176). Göttingen: Hogrefe.

Hemmer, I. (2010). Erforschung von Schülerinteressen als wichtige fachdidaktische Aufgabe - ein Überblick über den Stand der Forschung in Geographie und Geoscience. In I. Hemmer \& M. Hemmer (Hrsg.), Schülerinteresse an Themen, Regionen und Arbeitsweisen des Geographieunterrichts. Ergebnisse der empirischen Forschung und deren Konsequenzen für die Unterrichtspraxis (S. 27-61). Weingarten: Selbstverl. des Hochschulverbandes für Geographie und ihre Didaktik.

Hemmer, I., \& Hemmer, M. (2010a). Interesse von Schülerinnen und Schülern an einzelnen Themen, Regionen und Arbeitsweisen des Geographieunterrichts - ein Vergleich zweier empirischer Studien aus den Jahren 1995 und 2005. In I. Hemmer \& M. Hemmer (Hrsg.), Schülerinteresse an Themen, Regionen und Arbeitsweisen des Geographieunterrichts. Ergebnisse der empirischen Forschung und deren Konsequenzen für die Unterrichtspraxis (S. 65-145). Weingarten: Selbstverl. des Hochschulverbandes für Geographie und ihre Didaktik.

Hemmer, I., \& Hemmer, M. (2010b). Interesse von Schülerinnen und Schülern an geowissenschaftlichen Themen und Arbeitsweisen - Zur Bedeutung der Kontexte. In I. Hemmer \& M. Hemmer (Hrsg.), Schülerinteresse an Themen, Regionen und Arbeitsweisen des Geographieunterrichts. Ergebnisse der empirischen Forschung und deren Konsequenzen für die Unterrichtspraxis (S. 223-235). Weingarten: Selbstverl. des Hochschulverbandes für Geographie und ihre Didaktik.

Hemmer, I., \& Hemmer, M. (2017). Teachers' interests in geography topics and regions-how they differ from students' interests? Empirical findings. Review of International Geographical Education Online (RIGEO), 7(1), 9-23. 
Hemmer, I., Hemmer, M., Bayrhuber, H., Häußler, P., Hlawatsch, S., Hoffmann, L., \& Raffel-Siefer, M. (2005). Interesse von Schülerinnen und Schülern an geowissenschaftlichen Themen. Ergebnisse einer Interessenstudie im Rahmen des Projekts „Forschungsdialog System Erde" unter besonderer Berücksichtigung des Geographieunterrichts. Geographie und ihre Didaktik, 33(2), 57-72.

Hemmer, M., Hemmer, I., Warnke, M., \& Zietzsch, P. (2019). Welche Themen interessieren Schülerinnen und Schüler des Gymnasiums im Geographieunterricht? Ausgewählte Ergebnisse einer empirischen Untersuchung in Nordrhein-Westfalen. Bd. 93 (S. 34-41).

Hemmer, M., Rahner, M., \& Schuler, S. (2011). Naturtrisiken im Geographieunterricht - ausgewählte Ergebnisse empirischer Studien zur Schülerperspektive, didaktische Konsequenzen und Forschungsperspektiven. Geographie und ihre Didaktik, 39(1), $1-24$.

Hlawatsch, S., Lücken, M., Hansen, K.-H., Fischer, M., \& Bayrhuber, H. (2005). Forschungsdialog: System Erde. Schlussbericht. Kiel. http://archiv.ipn.uni-kiel.de/System_Erde/Schlussbericht20_12_ 05-EF.pdf. Zugegriffen: 12. Febr. 2021.

Hoffmann, L., Häußler, P., \& Lehrke, M. (1998). Die IPN-Interessenstudie Physik. Kiel: IPN.

Höhnle, S. (2014). Online-gestützte Projekte im Kontext Globalen Lernens im Geographieunterricht. Empirische Rekonstruktion internationaler Schülerperspektiven. Münster: Monsenstein und Vannerdat.

Intergovernmental Panel on Climate Change (IPCC) (2014). Klimaänderungen 2014. Synthesebericht

Jacobeit, J. (2007). Zusammenhänge und Wechselwirkungen im Klimasystem. In W. Endlicher \& F.-W. Gerstengarbe (Hrsg.), Der Klimawandel. Einblicke, Rückblicke und Ausblicke (S. 1-16). Potsdam: Potsdam-Institut für Klimafolgenforschung e.V.

Jöreskog, K. G. (1999). How large can a Standarized coefficient be? http://www.statmodel.com/download/Joreskog.pdf. Zugegriffen: 6. Juli 2020.

Kortland, K. (2005). Physics in personal, social and scientific contexts. A retrospective view on the Dutch physics curriculum development project. In P. Nentwig \& D. J. Waddington (Hrsg.), Making it relevant. Context based learning of science (S. 67-89). Münster: Waxmann.

Krapp, A. (1992a). Das Interessenkonstrukt. Bestimmungsmerkmale der Interessenhandlung und des individuellen Interesses aus Sicht einer Person-Gegenstands-Konzeption. In A. Krapp \& M. Prenzel (Hrsg.), Interesse, Lernen, Leistung. Neuere Ansätze der pädagogisch-psychologischen Interessenforschung (S. 297-329). Münster: Aschendorff.

Krapp, A. (1992b). Interesse, Lernen und Leistung. Neuere Forschungsansätze in der Pädagogischen Psychologie. Zeitschrift für Pädagogik, 38(5), 747-770.

Krapp, A. (1992c). Konzepte und Forschungsansätze zur Analyse des Zusammenhangs von Interesse, Lernen und Leistung. In A. Krapp \& M. Prenzel (Hrsg.), Interesse, Lernen, Leistung. Neuere Ansätze der pädagogisch-psychologischen Interessenforschung (S. 9-52). Münster: Aschendorff.

Krapp, A. (2010a). Die Bedeutung von Interessen für die Lernmotivation und das schulische Lernen - eine Einführung. In I. Hemmer \& M. Hemmer (Hrsg.), Schülerinteresse an Themen, Regionen und Arbeitsweisen des Geographieunterrichts. Ergebnisse der empirischen Forschung und deren Konsequenzen für die Unterrichtspraxis (S. 9-26). Weingarten: Selbstverl. des Hochschulverbandes für Geographie und ihre Didaktik.

Krapp, A. (2010b). Interesse. In D. H. Rost (Hrsg.), Handwörterbuch Pädagogische Psychologie (S. 311-323). Weinheim: Beltz.

Krapp, A., \& Ryan, R.M. (2002). Selbstwirksamkeit und Lernmotivation. Eine kritische Betrachtung der Theorie von Bandura aus der Sicht der Selbstbestimmungstheorie und der pädagogischpsychologischen Interessentheorie. In M. Jerusalem \& D. Hopf
(Hrsg.), Selbstwirksamkeit und Motivationsprozesse in Bildungssituationen (S. 54-82). Weinheim: Beltz.

Lüdtke, O., Robitzsch, A., Trautwein, U., \& Köller, O. (2007). Umgang mit fehlenden Werten in der psychologischen Forschung. Psychologische Rundschau, 58(2), 103-117.

Mandl, H., \& Kopp, B. (2005). Situated learning: theories and models. In P. Nentwig \& D. J. Waddington (Hrsg.), Making it relevant. Context based learning of science (S. 15-34). Münster: Waxmann.

Mikelskis-Seifert, S., \& Duit, R. (2007). Physik im Kontext. Innovative Unterrichtsansätze für den Schulalltag. Der mathematische und naturwissenschaftliche Unterricht (MNU), 60(5), 265-273.

Moschopoulos, P.G. (1983). On a new transformation to normality. Communications in Statistics-Theory and Methods, 12(16), 1873-1878. https://www.tandfonline.com/doi/pdf/10.1080/03610 928308828577?needAccess=true, zuletzt geprüft am 15.07.2020.

Muckenfuß, H. (2004). Themen und Kontexte als Strukturelemente des naturwissenschaftlichen Unterrichts. Zu den Schwierigkeiten, systematisches Physiklernen zu organisieren. Physik und Didaktik in Schule und Hochschule, 2(3), 57-66.

Nentwig, P., \& Waddington, D. J. (Hrsg.). (2005). Making it relevant. Context based learning of science. IPN-UYSEG Conference in Science Education, Leibniz-Institute for Science Education; University of York. Münster: Waxmann.

Nentwig, P., Parchmann, I., Demuth, R., Gräsel, C., \& Ralle, B. (2005). Chemie im Kontext-From situated learning in relevant contexts to a systematic development of basic chemical concepts. In P. Nentwig \& D. J. Waddington (Hrsg.), Making it relevant. Context based learning of science (S. 155-173). Münster: Waxmann.

Oelgeklaus, H. (2012). Den Klimawandel unterrichten. Untersuchung zum Pedagogical Content Knowledge (PCK) von Lehrkräften zum Thema Klimawandel. Hamburg: Kovač.

van Oers, B. (1998). From context to contextualizing. Learning and Instruction, 8(6), 473-488.

Organisation for Economic Cooperation and Development (2006). Assessing scientific, reading and mathematical literacy. A framework for PISA 2006. Paris: OECD. Education and skills

Parchmann, I., \& Kuhn, J. (2018). Lernen im Kontext. In D. Krüger, I. Parchmann \& H. Schecker (Hrsg.), Theorien in der naturwissenschaftsdidaktischen Forschung (S. 193-208). Berlin, Heidelberg: Springer.

Rahmstorf, S., \& Schellnhuber, H.-J. (2019). Der Klimawandel. Diagnose, Prognose, Therapie. München: C.H.Beck.

Renninger, K. A., \& Hidi, S. (2019). Interest development and learning. In K. A. Renninger \& S. Hidi (Hrsg.), The Cambridge handbook of motivation and learning (S. 265-290). Cambridge: Cambridge University Press.

Rheinberg, F. (2010). Intrinsische Motivation und Flow-Erleben. In J. Heckhausen \& H. Heckhausen (Hrsg.), Motivation und Handeln (S. 365-387). Berlin, Heidelberg: Springer.

Ryan, R.M., \& Deci, E.L. (2004). Overview of self-determination theory: an organismic dialectical perspective. In E.L. Deci \& R. M. Ryan (Hrsg.), Handbook of self-determination research (S. 3-33). Rochester: University of Rochester Press.

Schendera, C.F. G. (2010). Clusteranalyse mit SPSS. Mit Faktorenanalyse. München: Oldenbourg.

Schuler, S. (2009). Schülervorstellungen zu Bedrohung und Verwundbarkeit durch den globalen Klimawandel. Geographie und ihre Didaktik, 37(1), 1-28.

Sekretariat der Ständigen Konferenz der Kultusminister der Länder in der Bundesrepublik Deutschland (KMK) (2005). Bildungsstandards im Fach Biologie für den Mittleren Schulabschluss. Beschluss vom 16.12.2004

Siegmund, A. (2008). Der Klimawandel - Eine aktuelle Bilanz globaler Prozesse und ihrer regionalen Folgen. UmweltWirtschaftsForum (uwf), 16(1), 3-11. 
Sjøberg, S., \& Schreiner, C. (2010). The ROSE project. Overview and key findings. Oslo: University of Oslo. https://roseproject.no/ network/countries/norway/eng/nor-Sjoberg-Schreiner-overview2010.pdf. Zugegriffen: 12. Febr. 2021.

Thomas, C.L., \& Kirby, L. A. J. (2020). Situational interest helps correct misconceptions: an investigation of conceptual change in university students. Instructional Science (Instr Sci), 48(3), 223-241.

Vogt, H. (2007). Theorie des Interesses und des Nicht-Interesses. In D. Krüger \& H. Vogt (Hrsg.), Theorien in der biologiedidaktischen Forschung. Ein Handbuch für Lehramtsstudenten und Doktoranden (S. 9-20). Berlin, Heidelberg: Springer.

van Vorst, H., Dorschu, A., Fechner, S., Kauertz, A., Krabbe, H., \& Sumfleth, E. (2015). Charakterisierung und Strukturierung von Kontexten im naturwissenschaftlichen Unterricht - Vorschlag ei- ner theoretischen Modellierung. Zeitschrift für Didaktik der Naturwissenschaften (ZfDN), 21(1), 29-39.

van Vorst, H., Fechner, S., \& Sumfleth, E. (2018). Unterscheidung von Kontexten für den Chemieunterricht. Zeitschrift für Didaktik der Naturwissenschaften (ZfDN), 24(1), 167-181.

Walter, O., \& Taskinen (2008). Naturwissenschaftsbezogene Motivationen und Kompetenzen von Schülerinnen und Schülern mit Migrationshintergrund in Deutschland: der Einfluss der Generation, der Herkunft und des Elternhauses. Zeitschrift für Erziehungswissenschaft (ZfE), 10(Sonderheft 10), 185-203.

Weinert, F.E. (2014). Vergleichende Leistungsmessung in Schulen eine umstrittene Selbstverständlichkeit. In F.E. Weinert (Hrsg.), Leistungsmessungen in Schulen (S. 17-31). Weinheim: Beltz. 